\title{
Treatment Response of Patients with Erythrodermic Psoriasis after Switching to Guselkumab
}

\author{
Cheng-Ying Chiang (D) · Tsen-Fang Tsai (D
}

Received: October 21, 2020 / Published online: January 4, 2021

(c) The Author(s) 2021

\begin{abstract}
Introduction: Erythrodermic psoriasis (EP) is a rare variant of psoriasis. Due to its rarity, evidence supporting its treatment is limited. Patients with erythrodermic psoriasis often respond less favorably to conventional treatment and have lower biologic drug survival compared with patients with plaque-type psoriasis. Guselkumab is an anti-interleukin-23 (IL23) monoclonal antibody and has shown excellent and sustained treatment effect in moderate-to-severe plaque-type psoriasis. Until now, there is only one phase III open-label study reporting the effectiveness of guselkumab in this patient group.

Methods: Patients with chronic erythrodermic psoriasis who had been commenced on guselkumab were included in this retrospective study. All except one of our patients had plaque-type psoriasis prior to EP, and they met the criteria for EP before use of guselkumab. Due to inadequate response to previous treatment, including biologics, they were switched to guselkumab treatment. There was no washout period. They
\end{abstract}

C.-Y. Chiang · T.-F. Tsai ( $₫)$

Department of Dermatology, National Taiwan

University College of Medicine, National Taiwan

University Hospital, Taipei, Taiwan

e-mail: tftsai@yahoo.com received guselkumab $100 \mathrm{mg}$ at weeks 0 and 4, then every 8 weeks until at least week 28. Data were collected for patient demographics, Patient Area and Severity Index (PASI), and previous treatments.

Results: A total of 13 patients (12 male, 1 female) were included. The mean PASI improvement at weeks $4,12,20$, and 28 was $37.5 \%, 60.9 \%, 67.5 \%$, and $64.7 \%$, respectively. The percentage of PASI 75 responders at weeks $4,12,20$, and 28 was $15.4 \%, 38.5 \%, 53.9 \%$, and $46.2 \%$, respectively. At week 12, eight patients $(61.5 \%)$ could achieve PASI 50 response. These PASI 50 responders at week 12 showed sustained and improved effectiveness during follow-up.

Conclusion: Guselkumab is an effective biologic for treatment of moderate-to-severe plaque-type psoriasis, but the efficacy and drug survival are still compromised in EP patients. Our data describe the real-world experience of guselkumab treatment in this rare group of patients. Despite previous failure of other biologics, PASI response at week 12 is a good predictor of patient response and drug survival of guselkumab.

Keywords: Drug survival; Erythrodermic psoriasis; Guselkumab 


\section{Key Summary Points}

\section{Why carry out this study?}

Erythrodermic psoriasis (EP) is a rare variant of psoriasis. Due to its rarity, evidence supporting its treatment is limited.

To determine the efficacy of guselkumab in EP patients who were previously treated with other biologics or conventional treatment.

\section{What was learned from the study?}

The PASI 75 and PASI 90 response rates were lower in our EP case series than in the pivotal phase III studies which included patients with plaque-type psoriasis.

In our patients with EP, biologic naïvity and no history of secondary failure to biologic were good factors predicting guselkumab efficacy.

In our patients with EP, PASI response at week 12 seems to predict sustained drug survival.

\section{DIGITAL FEATURES}

This article is published with digital features, including a summary slide, to facilitate understanding of the article. To view digital features for this article go to https://doi.org/10.6084/ m9.figshare.13379132.

\section{INTRODUCTION}

Erythrodermic psoriasis (EP) is a rare and potentially fatal variant of psoriasis. EP was defined as psoriasis involving more than 80\% body surface area (BSA). Due to its low prevalence, evidence-based treatment choices are mainly from small case series or case report.
Patients with EP often showed limited improvement with conventional treatment and have shorter biologic drug survival compared with patients with plaque-type psoriasis [1-4]. Guselkumab is a fully human monoclonal antibody designed to bind to the p19 subunit of interleukin-23 (IL-23), thereby inhibiting the inflammatory cascade in psoriatic skin [5]. High and sustained efficacy has been demonstrated in pivotal studies in patients with chronic plaque-type psoriasis [6]. However, the only openlabel study, which led to approval for use of EP in Japan, included only 11 patients [7]. Herein, we report our experience in a series of patients with EP from a tertiary referral center in Taiwan.

\section{METHODS}

\section{Study Population and Treatment Protocol}

We reviewed medical records at a tertiary referral center in Taiwan and identified 13 patients with EP. All except one of our patients had plaque-type psoriasis before, and the mean \pm standard deviation (SD) duration of psoriasis was $24.2 \pm 13.3$ years. Two patients had fever, and another three had chills during erythrodermic state. Four patients, including the one who presented with erythroderma first, received skin biopsies and the histopathologic pictures were compatible with psoriasis. Other patients did not receive biopsy due to history of typical plaque-type psoriasis previously. They met the criteria for EP (BSA $\geq 80 \%$ ) before use of guselkumab. One patient reported amoxicillin and nonsteroidal antiinflammatory drugs (NSAIDs) as triggering factors; one patient considered upper respiratory tract infection as possible triggering factor; two patients considered seasonal change as trigger factor; two patients stated psychological stress as trigger factor. There were five patients with transient palmoplantar keratoderma during psoriasis aggravation in our case series.

All the patients did not have adequate response to conventional oral systemic agents and phototherapy, and most of them had secondary failure to multiple biologics. They were enrolled in our study and received guselkumab 
$100 \mathrm{mg}$ at weeks 0 and 4 , then every 8 weeks until at least week 28. There was no washout period. However, since our patients were switched from prior biologics or conventional systemic treatment, not every patient's involved skin lesions exceeded $80 \%$ BSA when they started guselkumab treatment. PASI response and treatment-emergent adverse events were evaluated by the same dermatologist at each visit. The study was approved by National Taiwan University Hospital Institutional Review Board (201904124RINC) and was performed in accordance with the Declaration of Helsinki of 1964 and its later amendments. Informed consent to participate in the study was obtained from all patients. There is no identifiable information in our paper.

\section{RESULTS}

A total of 13 patients [12 male (92.3\%), 1 female (7.7\%)] were included. Six (46.2\%) of the 13 patients had psoriatic arthritis. Mean \pm SD age was $50.5 \pm 15.3$ years. Mean \pm SD body weight was $71.0 \pm 13.5 \mathrm{~kg}$, and mean $\pm \mathrm{SD}$ body mass index was $24.6 \pm 4.1 \mathrm{~kg} / \mathrm{m}^{2}$. Mean \pm SD baseline Psoriasis Area and Severity Index (PASI) was $23.8 \pm 9.7$ (Table 1). Mean PASI improvement at weeks $4,12,20$, and 28 was $37.5 \%, 60.9 \%$,

Table 1 Summary of 13 cases of erythrodermic psoriasis

Patient demographics $^{\mathrm{a}}(n=13)$

Male sex, $n(\%)$

Age (years) $\pm S D$

Body weight $(\mathrm{kg}) \pm \mathrm{SD}$

BMI $\left(\mathrm{kg} / \mathrm{m}^{2}\right) \pm \mathrm{SD}$

History of psoriasis (years) $\pm \mathrm{SD}$

Psoriatic arthritis, $n(\%)$

Previous conventional systemic treatment, $n$ (\%)

Previous biologics use, $n(\%)$

Previous inadequate response to biologics, $n$ (\%)

Baseline PASI (absolute PASI before guselkumab) \pm SD

PASI improvement
$12(92.3 \%)$
$50.5 \pm 15.3$
$71.0 \pm 13.5$
$24.6 \pm 4.1$
$24.2 \pm 13.3$
$6(46.2 \%)$
$13(100 \%)$
$12(92.3 \%)$
$11(84.6 \%)$
$23.8 \pm 8.7$

\begin{tabular}{|c|c|c|c|c|}
\hline & Week 4 & Week 12 & Week $20^{b}$ & Week $28^{\mathrm{b}}$ \\
\hline Mean PASI response \pm SD & $37.5 \pm 30.1$ & $60.9 \pm 23.6$ & $67.5 \pm 30.0$ & $64.7 \pm 36.7$ \\
\hline Number of PASI responders $(n, \%)$ & Week 4 & Week 12 & Week $20^{b}$ & Week $28^{b}$ \\
\hline PASI 50 & $5(38.5 \%)$ & $8(61.5 \%)$ & $8(61.5 \%)$ & $8(61.5 \%)$ \\
\hline PASI 75 & $2(15.4 \%)$ & $5(38.5 \%)$ & $7(53.8 \%)$ & $6(46.2 \%)$ \\
\hline PASI 90 & 0 & $1(7.7 \%)$ & $3(23.1 \%)$ & $4(30.8 \%)$ \\
\hline PASI 100 & 0 & 0 & 0 & $2(15.4 \%)$ \\
\hline
\end{tabular}

BMI, body mass index; PASI, Psoriasis Area and Severity Index

a Values are $n(\%)$ or mean $\pm \mathrm{SD}$ (standard deviation)

b One patient discontinued guselkumab treatment at week 12 due to unsatisfactory efficacy 


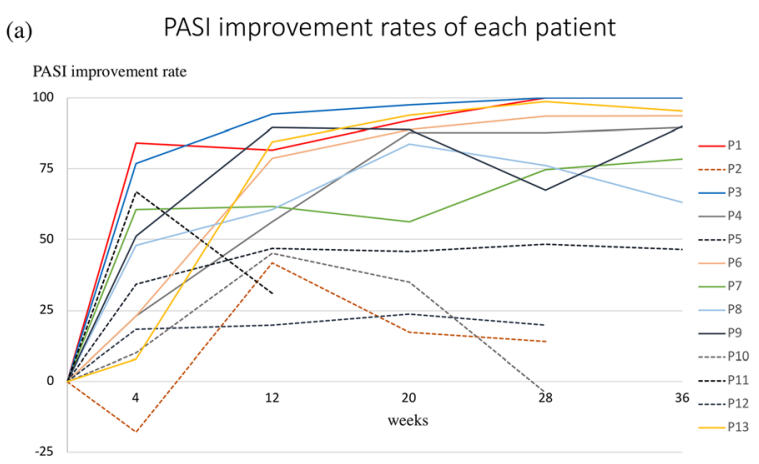

(b)

Mean PASI improvement rates of responders and non-responders Mean PASI improvement rate

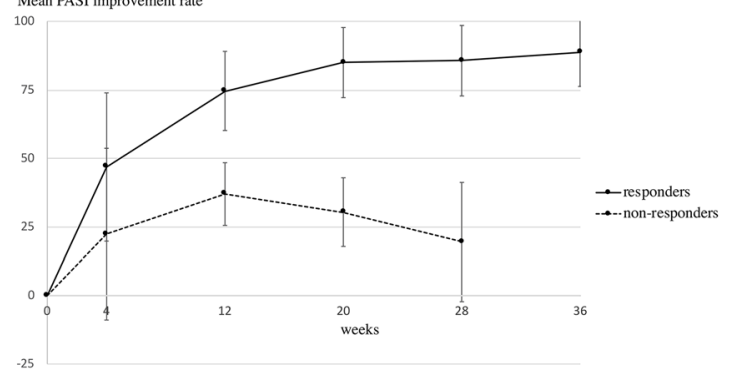

Fig. 1 a PASI improvement rates of each patient during guselkumab treatment. Different color lines represent the PASI response of different patients. Solid lines represent those patients who had PASI 50 response at week 12 . Dotted lines represent those patients who did not have PASI 50 response at week 12. Two patients had PASI 100 response at week 28 and week 36. One of the nonresponders discontinued guselkumab treatment because of unsatisfactory efficacy. b Mean PASI improvement of PASI 50 responders and nonresponders at week 12 during guselkumab treatment. For PASI 50 responders at week 12 , the mean \pm SD PASI improvement at week 4, 12, 20, 28 , and 36 was $46.9 \pm 27.1 \%, \quad 74.7 \pm 14.5 \%$, $85.0 \pm 12.7 \%, 85.7 \pm 12.8 \%$, and $88.8 \pm 12.5 \%$, respectively. For PASI 50 nonresponders at week 12, mean \pm SD PASI improvement at weeks 4, 12, 20, and 28 was $22.4 \pm 31.3 \%, \quad 37.0 \pm 11.5 \%, \quad 30.5 \pm 12.5 \%, \quad$ and $19.5 \pm 21.7 \%$, respectively. PASI, Patient Area and Severity Index; Patient, P

$67.5 \%$, and $64.7 \%$, respectively. The percentage of PASI 75 response at weeks $4,12,20$, and 28 was $15.4 \%, 38.4 \%, 53.8 \%$, and $46.2 \%$, respectively. The percentage of PASI 90 response at weeks $4,12,20$, and 28 was $0 \%, 7.7 \%, 23.1 \%$, and $30.8 \%$, respectively (Table 1 ).

The PASI improvement of each patient is shown in Fig. 1a. By week 12, PASI 50 response was seen in eight patients (61.5\%) (Fig. 1a, solid line). Most of these patients showed a trend of continuous PASI improvement through week 36 , after which the PASI score gradually became stable. One of them had psoriasis aggravation at week 28 (from PASI 89 response at week 20 to PASI 68 response at week 28) but achieved PASI 90 response again at week 36 . The other patient had psoriasis aggravation at week 36 (from PASI 76 at week 28 to PASI 63 at week 36).

For PASI 50 nonresponders at week 12 (five patients, 38.5\%) (Fig. 1a, dotted line), there was a decreasing trend in PASI improvement through week 28; one of them was prescribed cyclosporine from week 12, and four patients were switched to other biologics (one switched to brodalumab at week 12; the other three switched to adalimumab, brodalumab, and ixekizumab, respectively, at week 28).

No nasopharyngitis, headache, upper respiratory tract infection, or injection-site reaction was reported in our case series through week 36.

\section{DISCUSSION}

Patients with EP often have shorter biologic survival period than patients with plaque-type psoriasis. However, two reports have shown excellent and sustained treatment efficacy of guselkumab in EP $[7,8]$. In our case series, not all EP patients responded well to guselkumab. The PASI 75 and PASI 90 response rates were lower in our series than in the pivotal phase III studies which included patients with plaquetype psoriasis $[6,7]$. Most of our patients also showed a slower response compared with Sano's series of EP [7]. This lower and slower treatment response may be partly explained by the history of multiple prior biological failure in our patients $[3,9,10]$ and unstable disease course due to the lack of a washout period before receiving guselkumab. However, guselkumab still showed better drug survival compared with our prior experience of other biologics in a similar population, despite more prior biologic failures $[1,2]$.

At week 4, two patients (15.4\%) achieved more than PASI 75 improvement, including the only patient who was biologic naïve. The other 
early responder was treated with ustekinumab before and had no history of secondary failure. She was switched to guselkumab treatment due to the lack of ustekinumab in our hospital. These two early responders at week 4 achieved PASI 100 at week 28 (Fig. 1a). The other 11 patients $(84.6 \%)$ were resistant to at least one previous biological agent (etanercept: 5 patients; adalimumab: 6 patients; golimumab: 2 patients; ustekinumab: 8 patients; secukinumab: 9 patients; ixekizumab: 4 patients), and 9 of them $(69.2 \%)$ were resistant to more than one biological agent. Two, three, one, one, and two patients had failed two, three, four, five, and six prior biologics, respectively.

PASI 50 response at week 12 seems to predict long-term guselkumab drug survival. We divided the EP patients into responders and nonresponders according to their PASI response at week 12 (responders: PASI response $\geq 50$ at week 12; nonresponders: PASI response $<50$ at week 12). Responders (eight patients, 61.5\%) showed sustained and improved effectiveness during follow-up (Fig. 1b, mean \pm SD PASI improvement at weeks $4,12,20,28$, and 36 of $46.9 \pm 27.1 \%, \quad 74.7 \pm 14.5 \%, \quad 85.0 \pm 12.7 \%$, $85.7 \pm 12.8 \%$, and $88.8 \pm 12.5 \%$, respectively). Nonresponders showed shorter drug survival and quickly deteriorating PASI improvement after their peak response (Fig. $1 \mathrm{~b}$, mean \pm SD PASI improvement at weeks $4,12,20$, and 28 of $22.4 \pm 31.3 \%, \quad 37.0 \pm 11.5 \%, \quad 30.5 \pm 12.5 \%$, and $19.5 \pm 21.7 \%$, respectively).

Human leukocyte antigen (HLA)-Cw6 has been reported to affect the clinical efficacy of guselkumab in patients with moderate-to-severe psoriasis [11]. However, none of our patients had HLA-Cw6 allele. This may partly explain the slower PASI response in our patient series. However, the effect of HLA-Cw6 positivity on the effectiveness of guselkumab for psoriasis in Asian countries remains unidentified.

The limitations of the current study include a small patient population, lack of a control group, and statistical comparison. Despite these limitations, our data still describe an important real-world experience for this rare group of patients. Further studies are needed to identify the best treatment protocol for patients with EP who have failed prior biologics.

\section{CONCLUSION}

Guselkumab is an effective biologic for treatment of moderate-to-severe plaque-type psoriasis, but the efficacy and drug survival are still compromised in EP patients. In our patients with EP, biologic naïvity and no history of secondary failure to biologic were good factors predicting guselkumab efficacy. For those patients with history of secondary failure to other biologic, guselkumab is still effective in most patients. PASI response at week 12 seems to predict sustained drug survival.

\section{ACKNOWLEDGEMENTS}

We thank the participants of the study.

Funding. No funding or sponsorship was received for this study or publication of this article.

Authorship. All named authors meet the International Committee of Medical Journal Editors (ICMJE) criteria for authorship for this article, take responsibility for the integrity of the work as a whole, and have given their approval for this version to be published.

Disclosures. Cheng-Ying Chiang has nothing to disclose. Tsen-Fang Tsai is a member of the journal's editorial board.

Compliance with Ethics Guidelines. Compliance with Ethics Guidelines: The study was approved by National Taiwan University Hospital Institutional Review Board (201904124RINC) and was performed in accordance with the Helsinki Declaration of 1964 and its later amendments. Informed consent to participant in the study was obtained from all patients. There was no identifiable information in our paper.

Data Availability. The datasets generated during and/or analysed during the current study are available from the corresponding author on reasonable request. 
Open Access. This article is licensed under a Creative Commons Attribution-NonCommercial 4.0 International License, which permits any non-commercial use, sharing, adaptation, distribution and reproduction in any medium or format, as long as you give appropriate credit to the original author(s) and the source, provide a link to the Creative Commons licence, and indicate if changes were made. The images or other third party material in this article are included in the article's Creative Commons licence, unless indicated otherwise in a credit line to the material. If material is not included in the article's Creative Commons licence and your intended use is not permitted by statutory regulation or exceeds the permitted use, you will need to obtain permission directly from the copyright holder. To view a copy of this licence, visit http://creativecommons.org/licenses/by$\mathrm{nc} / 4.0 /$.

\section{REFERENCES}

1. Wang TS, Tsai TF. Clinical experience of ustekinumab in the treatment of erythrodermic psoriasis: a case series. J Dermatol. 2011;38:1096-9.

2. Weng HJ, Wang TS, Tsai TF. Clinical experience of secukinumab in the treatment of erythrodermic psoriasis: a case series. Br J Dermatol. 2018;178: 1439-40.

3. Egeberg A, Bryld LE, Skov L. Drug survival of secukinumab and ixekizumab for moderate-to- severe plaque psoriasis. J Am Acad Dermatol. 2019;81:173-8.

4. Chiu TS, Tsai TF. Treatment response in patients with moderate to severe psoriasis who had inadequate response to prior secukinumab. Dermatologica Sinica. 2019;37:129-33.

5. Levin AA, Gottlieb AB. Specific targeting of interleukin-23p19 as effective treatment for psoriasis. J Am Acad Dermatol. 2014;70:555-61.

6. Nakamura M, Lee K, Jeon C, et al. Guselkumab for the treatment of psoriasis: a review of phase III trials. Dermatol Ther (Heidelb). 2017;7:281-92.

7. Sano S, Kubo H, Morishima H, et al. Guselkumab, a human interleukin-23 monoclonal antibody in Japanese patients with generalized pustular psoriasis and erythrodermic psoriasis: efficacy and safety analyses of a 52-week, phase 3, multicenter, openlabel study. J Dermatol. 2018;45:529-39.

8. Megna M, Ruggiero A, Camela E, Fabbrocini G, et al. A case of erythrodermic psoriasis successfully treated with guselkumab. Dermatol Ther. 2020;33: e13238.

9. Ger TY, Huang YH, Hui RC, et al. Effectiveness and safety of secukinumab for psoriasis in real-world practice: analysis of subgroups stratified by prior biologic failure or reimbursement. Ther Adv Chronic Dis. 2019;10:2040622319843756.

10. Lo Y, Chiu HY, Tsai TF. Clinical features and genetic polymorphism in Chinese patients with erythrodermic psoriasis in a single dermatologic clinic. Mol Diagn Ther. 2020;24:85-93.

11. Liu $X$, DePrimo $S$, Chen $Y$, et al. Association between HLA-Cw6 status and response to guselkumab in patients with moderate to severe plaque psoriasis. J Investig Dermatol. 2018;138:S76. 